\title{
Atypical functional connectivity in adolescents and adults with persistent and remitted ADHD during a cognitive control task
}

Giorgia Michelini $\mathbb{D}^{1,2}$, Joseph Jurgiel ${ }^{3}$, loannis Bakolis${ }^{4}$, Celeste H. M. Cheung ${ }^{1}$, Philip Asherson ${ }^{1}$, Sandra K. Loo ${ }^{3}$, Jonna Kuntsi ${ }^{1}$ and Iman Mohammad-Rezazadeh ${ }^{3,5}$

\begin{abstract}
We previously provided initial evidence for cognitive and event-related potential markers of persistence/remission of attention-deficit/hyperactivity disorder (ADHD) from childhood to adolescence and adulthood. Here, using a novel brain-network connectivity approach, we aimed to examine whether task-based functional connectivity reflects a marker of ADHD remission or an enduring deficit unrelated to ADHD outcome. High-density EEG was recorded in a follow-up of 110 adolescents and young adults with childhood ADHD (87 persisters, 23 remitters) and 169 typically developing individuals during an arrow-flanker task, eliciting cognitive control. Functional connectivity was quantified with network-based graph-theory metrics before incongruent (high-conflict) target onset (pre-stimulus), during target processing (post-stimulus) and in the degree of change between pre-stimulus/post-stimulus. ADHD outcome was examined with parent-reported symptoms and impairment using both a categorical (DSM-IV) and a dimensional approach. Graph-theory measures converged in indicating that, compared to controls, ADHD persisters showed increased connectivity in pre-stimulus theta, alpha, and beta and in post-stimulus beta (all $p<.01$ ) and reduced prestimulus/post-stimulus change in theta connectivity $(p<.01)$. In the majority of indices showing ADHD persister-control differences, ADHD remitters differed from controls (all $p<.05$ ) but not from persisters. Similarly, connectivity measures were unrelated to continuous outcome measures of ADHD symptoms and impairment in participants with childhood ADHD. These findings indicate that adolescents and young adults with persistent and remitted ADHD share atypical over-connectivity profiles and reduced ability to modulate connectivity patterns with task demands, compared to controls. Task-based functional connectivity impairments may represent enduring deficits in individuals with childhood ADHD irrespective of diagnostic status in adolescence/young adulthood.
\end{abstract}

Correspondence: Giorgia Michelini (Giorgia.Michelini@stonybrookmedicine. edu) or Jonna Kuntsi (Jonna.Kuntsi@kcl.ac.uk)

'Social, Genetic and Developmental Psychiatry Centre, Institute of Psychiatry, Psychology and Neuroscience, King's College London, London, UK

${ }^{2}$ Department of Psychiatry and Behavioral Health, State University New York

(SUNY) Stony Brook University, Stony Brook, NY, USA

Full list of author information is available at the end of the article.

These authors contributed equally: Jonna Kuntsi, Iman Mohammad-Rezazadeh

\section{Introduction}

A coherent communication between brain regions organized in large-scale neural systems, or brain functional connectivity, is thought to have a key role in cognition and behavior ${ }^{1-4}$. Accumulating evidence suggests that atypical connectivity may be implicated in neurodevelopmental disorders ${ }^{5-7}$, such as attention-deficit/ hyperactivity disorder (ADHD). In recent years, the study of functional connectivity in ADHD has contributed to

\section{(c) The Author(s) 2019}

(c) (i) Open Access This article is licensed under a Creative Commons Attribution 4.0 International License, which permits use, sharing, adaptation, distribution and reproduction cc) in any medium or format, as long as you give appropriate credit to the original author(s) and the source, provide a link to the Creative Commons license, and indicate if changes were made. The images or other third party material in this article are included in the article's Creative Commons license, unless indicated otherwise in a credit line to the material. If material is not included in the article's Creative Commons license and your intended use is not permitted by statutory regulation or exceeds the permitted use, you will need to obtain permission directly from the copyright holder. To view a copy of this license, visit http://creativecommons.org/licenses/by/4.0/. 
the development of neurobiological models highlighting the role of multiple large-scale neural systems in the $\operatorname{disorder}^{8-13}$. These include the frontal-parietal network, the ventral-attentional network, and the default-mode network (DMN), involved in executive control, attentional processes, and introspective resting states, respectively ${ }^{8,9,14}$. In particular, it has been hypothesized that ADHD symptomatology may arise from a deviation from neurotypical synchronization and interaction within and between these large-scale networks during brain development $^{8,12,15,16}$. Characterizing the atypical patterns of whole-brain functional connectivity across development may thus provide new insights into ADHD neurobiology.

Most studies to date have investigated functional connectivity in ADHD using resting-state functional magnetic-resonance imaging (fMRI) and reported both reduced $^{14,16-19}$ and increased ${ }^{5,20-26}$ connectivity, for example, within and between the DMN and executive network. Task-based fMRI connectivity studies, which may allow for a more direct characterization of connectivity underlying impaired cognition and behavior ${ }^{27,28}$, have further shown not only hypo-connectivity in frontostriato-cerebellar networks during sustained attention ${ }^{29}$ and inhibition ${ }^{30-32}$ but also hyper-connectivity within the $\mathrm{DMN}^{30}$ and between networks of reward-cognitive control integration ${ }^{33}$ in ADHD. The inconsistencies across previous findings may arise from relatively small samples in most studies and differences in methodology (e.g., region-of-interest vs whole-brain analyses) ${ }^{12,34}$. In addition, fMRI connectivity measures synchronicity between precisely localized networks $\mathrm{s}^{5,8,35}$ but may not fully capture synchronization between faster brain oscillations underlying fast-changing processes during cognitive tasks.

Investigating functional connectivity using the subsecond temporal resolution of electroencephalography (EEG) instead allows for the measurement of a wider range of brain oscillatory phenomena, including transient changes in connectivity during cognition and behavior $^{36,37}$. Theta, alpha, and beta oscillations during cognitive tasks ${ }^{4,38-40}$, such as flanker tasks ${ }^{41-45}$, have been implicated in processes engaging top-down control networks that require coherent activity between cortically distributed regions. While most EEG connectivity studies on ADHD to date have focused on resting states ${ }^{46-48}$, available task-based connectivity studies in children and adolescents with ADHD indicate not only hyperconnectivity in alpha ${ }^{49}$ and beta ${ }^{37}$ oscillations during attentional tasks but also reduced fronto-parietal theta-alpha connectivity during a flanker task ${ }^{45,50}$. Yet, most EEG connectivity studies on ADHD have used connectivity metrics contaminated by volume-conduction artifacts (i.e., the spreading and mixing of multiple brain sources at the scalp). This methodological limitation can produce inflated connectivity estimates significantly affecting case-control differences ${ }^{51,52}$ and warrants further investigation with metrics uncontaminated by this issue. Furthermore, network approaches based on graph theory have been recently applied to characterize functional connectivity between large-scale brain networks and identify connectivity alterations ${ }^{2,5,53}$. Initial graphtheory evidence from one task-based EEG study showed atypical functional connectivity in children with $\mathrm{ADHD}^{54}$, but no study to date has been conducted on adolescents or adults ${ }^{4,38-45}$.

Despite the hypothesis that a deviation from neurotypical large-scale connectivity profiles across development may be implicated in $\mathrm{ADHD}^{8,12,15,16}$, little is known on how functional connectivity alterations map onto ADHD developmental outcomes. Longitudinal studies show that ADHD persists, in full or in partial remission, in the majority of adolescents and adults clinically diagnosed in childhood, while a proportion of individuals remit across development ${ }^{55,56}$. Remission of ADHD may be explained in light of compatible neurodevelopmental models ${ }^{57,58}$, which posit that remission may underlie (1) a "normalization" of neural processes (markers of remission) that improve concurrently with clinical symptoms and impairment, whereby individuals with remitted ADHD (ADHD "remitters") converge toward neurotypical individuals but diverge from individuals with persistent ADHD (ADHD "persisters") ${ }^{11,59-61}$; and (2) enduring deficits that are unrelated to the clinical outcome, remaining impaired in both ADHD remitters and persisters compared to neurotypical controls ${ }^{11,62}$. The identification of such processes is important for elucidating the neurobiological mechanisms underlying remission/persistence and may point to candidate biomarkers for the development of new interventions. It has been hypothesized that improvements across development in higherlevel executive functions would underlie ADHD remission, while persisting impairments in lower-level processes would be displayed regardless of later clinical outcome $^{11}$. Most studies to date, however, found that cognitive performance indices of executive functioning do not distinguish between ADHD persisters and remitters and are thus insensitive to ADHD outcomes ${ }^{60,61,63-66}$. In line with these studies, our recent follow-up study of adolescents and young adults with childhood ADHD show, using a range of attentional, vigilance, and executive paradigms, that cognitive and event-related potential (ERP) markers of executive control (inhibition, working memory, conflict monitoring N2) were insensitive to ADHD outcome ${ }^{60,61,67}$. Instead, cognitive-EEG measures of preparation-vigilance (e.g., reaction-time variability [RTV], target-P3) and error detection (e.g., error-related negativity [ERN] and positivity [Pe]) were markers of remission, distinguishing ADHD remitters from persisters $^{60,61,67}$. 
The investigation of task-based functional connectivity across distributed brain networks may provide new insights into the neural pathways of ADHD persistence/ remission. The only available task-based connectivity study on persistence/remission reported lower fMRI fronto-thalamic connectivity during response preparation in ADHD persisters compared to remitters and controls during a cued reaction-time task ${ }^{68}$. Despite the complementary benefit of EEG in investigating functional connectivity during cognitive processes ${ }^{36}$, no study to date has examined EEG connectivity in adult ADHD or in relation to remission/persistence.

In the present EEG study, we aimed to investigate brain functional connectivity during a cognitive control task, the arrow flanker task, in adolescents and adults with childhood ADHD and neurotypical controls. We previously reported that, during incongruent (high-conflict) trials of this task, the N2 index of conflict monitoring and cognitive performance measures were insensitive to ADHD outcomes, while error-related ERPs were markers of remission ${ }^{61}$. In this new in-depth analysis of the data presented in our previous study ${ }^{61}$, we sought to take a whole-brain approach to test whether functional connectivity before and during the processing of incongruent stimuli, measured with graph-theory and connectivity metrics not contaminated by volume conduction, is atypical in persistent ADHD, and whether it represents a marker of ADHD remission or an enduring deficit. We hypothesized that both ADHD persisters and remitters would display functional connectivity alterations compared to neurotypical individuals during this task evoking high levels of cognitive control, consistent with most studies examining cognitive and EEG markers of executive processes and ADHD remission ${ }^{60,61,63-65}$.

\section{Methods \\ Sample}

The sample consisted of 279 participants who were followed up on average 5.8 years $(\mathrm{SD}=1.1)$ after assessments in childhood ${ }^{69}$, including 110 adolescents and young adults who met Diagnostic and Statistical Manual of Mental Disorders, Fourth Edition (DSM-IV) criteria for combined-type ADHD in childhood (10 sibling pairs and 90 singletons) and 169 control participants (76 sibling pairs and 17 singletons) ${ }^{60,70}$. Participants with ADHD were initially recruited from specialized ADHD clinics and controls from schools in the $\mathrm{UK}^{69}$. Exclusion criteria at both assessments were: intelligence quotient (IQ) $<70$, autism, epilepsy, brain disorders, and any genetic/medical disorder associated with externalizing behaviors that might mimic ADHD. Among those with childhood ADHD, at follow-up 87 (79\%) continued to meet clinical (DSM-IV) levels of ADHD symptoms and impairment (ADHD persisters), while 23 (21\%) were below the clinical cut-off (ADHD remitters) ${ }^{71}$ (see "ADHD diagnosis" below). Among ADHD remitters, 14 displayed $\geq 5$ symptoms of inattention or hyperactivity-impulsivity but did not show functional impairment. Participants attended a single research session for clinical, IQ and cognitive-EEG assessments. An estimate of IQ was derived with the vocabulary and block design subtests of the Wechsler Abbreviated Scale of Intelligence $^{72}$. ADHD persisters, remitters, and controls did not differ in age, but there were significantly more males in the remitted group than in the other two groups, with no females among ADHD remitters (Table 1) ${ }^{60,61}$. ADHD persisters showed lower IQ compared to remitters and controls ${ }^{60,71}$. Among participants with childhood ADHD, 47\% were on drug treatment at follow-up, but the proportion of participants on medication did not differ between ADHD persisters and remitters $\left(\chi^{2}=1.95\right.$, $p=.16)^{60}$. A 48-h ADHD medication-free period was required before assessments. Parents of all participants gave informed consent following procedures approved by the London-Surrey Borders Research Ethics Committee (09/H0806/58).

Table 1 Sample demographics divided by group, with tests for differences between ADHD persisters, remitters, and controls

\begin{tabular}{|c|c|c|c|c|c|c|c|}
\hline & \multirow[t]{2}{*}{ ADHD-R $(n=23)$} & \multirow[t]{2}{*}{ ADHD-P $(n=87)$} & \multirow[t]{2}{*}{ Ctrl $(n=169)$} & \multicolumn{4}{|c|}{ Group comparison } \\
\hline & & & & $p$ & $\begin{array}{l}\text { Ctrl vs ADHD-P } \\
p\end{array}$ & $\begin{array}{l}\text { Ctrl vs ADHD-R } \\
p\end{array}$ & $\begin{array}{l}\text { ADHD-P vs ADHD-R } \\
p\end{array}$ \\
\hline Gender, M:F & $23: 0$ & $72: 15$ & $129: 40$ & $.02^{*}$ & .24 & $<.01^{* *}$ & $.03^{*}$ \\
\hline Age, mean (SD) & $18.89(3.06)$ & $18.27(3.03)$ & $18.77(2.19)$ & .15 & - & - & - \\
\hline $\mathrm{IQ}$, mean $(S D)$ & 104.57 (13.63) & $96.20(15.33)$ & $109.98(12.42)$ & $<.01^{* *}$ & $<.01^{* *}$ & .10 & $.02^{*}$ \\
\hline
\end{tabular}

Notes: Group differences on gender were tested via Chi-square test; group differences on age and IQ were tested with linear regressions. Group differences in gender, age, and IQ were previously reported in other papers on this sample ${ }^{60,61}$

$A D H D$ attention-deficit hyperactivity disorder, $A D H D-P$ ADHD persisters, $A D H D-R$ ADHD remitters, Ctrl Control group, $F$ number of females, $M$ number of males ${ }^{* *} p<.01$; ${ }^{*} p<.05$ 


\section{ADHD diagnosis}

The Diagnostic Interview for ADHD in Adults (DIVA) ${ }^{73}$ was conducted by trained researchers with parents of the ADHD probands to assess DSM-IV-defined ADHD presence and persistence of the 18 ADHD symptoms. Evidence of impairment commonly associated with ADHD was assessed with the Barkley's functional impairment scale $(B F I S)^{74}$. Parent-reported DIVA and impairments were used to determine ADHD status, as these were validated against objective markers (cognitive performance and EEG measures) in this sample, whereas the same objective markers showed limited agreement with self-reported $\mathrm{ADHD}^{75}$. Childhood ADHD participants were classified as "affected" at follow-up (i.e., ADHD persisters) if they showed $\geq 6$ items in either the inattention or hyperactivity-impulsivity domains on the DIVA and $\geq 2$ areas of impairments on the BFIS; they were classified as remitters otherwise. ADHD outcome was measured using a categorical definition of persistence based on diagnosis (i.e., meeting DSM-IV ADHD diagnostic criteria at follow-up), as well as a dimensional approach based on continuous levels of symptoms of ADHD and impairments, to assess ADHD severity.

\section{Task}

The task was an adaptation of the Eriksen Flanker paradigm designed to increase cognitive $\operatorname{load}^{76}$. In each trial, a central fixation mark was replaced by a target arrow (a black $18 \mathrm{~mm}$ equilateral triangle). Participants had to indicate whether this arrow pointed toward the left or right by pressing corresponding response buttons with their left or right index fingers. Two flanker arrows identical in shape and size to the target appeared $22 \mathrm{~mm}$ above and below the center of the target arrow $100 \mathrm{~ms}$ before each target. Both flankers either pointed in the same (congruent) or opposite (incongruent) direction to the target. Cognitive control and conflict monitoring are maximal during incongruent trials. When the target appeared, both target and flankers remained on the screen for $150 \mathrm{~ms}$, with a new trial every $1650 \mathrm{~ms}$. Two-hundred congruent and 200 incongruent trials were arranged in 10 blocks of 40 trials. Only incongruent trials were considered in the present in-depth analysis of the data included in our previous study ${ }^{61}$, as this high-conflict condition has proven more sensitive to ADHD-control differences in previous ERP analyses in this ${ }^{61}$ and other $^{76,77}$ ADHD samples. For further details, see Supplementary Material.

\section{EEG recording and processing}

The EEG was recorded from a 62-channel extended 10-20 system (Brain Products, GmbH, Munich, Germany), using a $500-\mathrm{Hz}$ sampling rate, impedances under $10 \mathrm{k} \Omega$, and recording reference at FCz. The electro- oculograms were recorded from electrodes above and below the left eye and at the outer canthi. Raw EEG recordings were down-sampled to $256 \mathrm{~Hz}$, re-referenced to the average of all electrodes (turning $\mathrm{FCz}$ into an active channel), and filtered using Butterworth band-pass filters $(0.10-30 \mathrm{~Hz}, 24 \mathrm{~dB} / \mathrm{oct})$. All trials were visually inspected and sections containing electrical or movement artifacts were removed manually. Ocular artifacts were identified using Independent Component Analysis ${ }^{78}$. Sections of data containing artifacts $> \pm 100 \mu \mathrm{V}$ or with a voltage step $\geq 50 \mu \mathrm{V}$ were automatically rejected. The artifact-free data were segmented in epochs between -650 and $1000 \mathrm{~ms}$ stimulus-locked to incongruent stimuli. Both trials with correct and incorrect responses were examined ${ }^{61}$. Only data containing $\geq 20$ clean segments for condition were included in analyses, leaving 271 participants (83 ADHD persisters, 22 remitters, 166 controls) for correctlyresponded trials and 240 (75 ADHD persisters, 20 remitters, 145 controls) for incorrectly-responded trials.

\section{Connectivity analysis Calculation of functional connectivity}

We calculated functional brain connectivity using the imaginary part of coherence (iCoh) ${ }^{51,79,80}$. This measure was chosen to ignore spurious connections between brain signals caused by volume conduction, which can substantially limit the ability to measure functional associations using EEG channels. iCoh captures the noninstantaneous connectivity between brain activities from EEG channels that are phase-lagged (i.e., delay-based) ${ }^{81,82}$. Since volume conduction affects multiple scalp channels with near-zero phase delays, connectivity measured with iCoh is not contaminated by near-instantaneous artifacts of volume conduction. iCoh was measured by isolating the imaginary part of the complex number phase coherence between two signals of same frequency ${ }^{51}$, estimated by calculating their cross-spectrum for each time point with Fast Fourier Transforms using the EEGLAB "newcrossf" function ${ }^{83}$ in Matlab (The Math Works Inc., Natick, MA, USA). iCoh is measured on a scale between 0 and 1 . When two signals at the same frequency have identical phase values, possibly due to volume conduction artifacts, $\mathrm{iCoh}=0$. Instead, if two signals are phase lagged, $\mathrm{iCoh}>0^{51}$. Values of iCoh for all possible electrode pairs $(62 \times 62)$ were computed in the theta $(4-8 \mathrm{~Hz})$, alpha $(8-12 \mathrm{~Hz})$, and beta $(12-20 \mathrm{~Hz})$ bands (Supplementary Fig. 1), which have previously been implicated in cognitive processes engaging top-down control networks requiring coherent activity between brain areas ${ }^{4,38,39}$, such as the fronto-parietal network ${ }^{84-87}$.

\section{Graph-theory metrics}

The high multi-dimensionality of the iCoh measures was disentangled with a graph-theory approach, which 
allows one to derive global network-based measures and describe functional associations in terms of network properties $^{2,88,89}$. Graph theory is based on mathematical algorithms to quantify the relationships ("edges") between brain signals from EEG channels, representing the "nodes" of a network. Unthresholded weighted iCoh matrices were used, in line with previous studies ${ }^{7,90-92}$, where each edge is equivalent to the measured iCoh of two electrodes to preserve essential information of a network structure ${ }^{2,93,94}$. Graph-theory metrics measure the degree of network segregation (i.e., the tendency of brain regions to form local clusters with dense functional interconnections) and network integration and efficiency (i.e., the capacity of the network to become interconnected and efficiently exchange information between brain regions $)^{2,95}$. The following commonly used graph measures were calculated ${ }^{7,54,91,93,96}$ : average clustering coefficient (the probability of neighboring nodes of being interconnected, forming densely inter-connected clusters); global efficiency (how efficient the network is in transferring information); and characteristic path length and diameter (respectively, the average number of edges along the shortest paths and the largest possible distance, between all possible pairs of nodes). Graph-theory metrics were computed with the Brain Connectivity ${ }^{47}$ and BioNeCT (https://sites.google.com/site/bionectweb/home; ref. ${ }^{3}$ ) toolboxes. In order to examine the modulation of functional connectivity profiles with different conditions and correct vs incorrect performance in this task, we computed connectivity metrics before target (pre-stimulus; -500 to $0 \mathrm{~ms}$ ) and during target processing (post-stimulus; 0 to $500 \mathrm{~ms}$ ), as well as separately for correctly- and incorrectly-responded trials.

\section{Statistical analyses \\ Categorical analysis based on diagnostic status}

Connectivity metrics were examined with randomintercept linear models (i.e., multilevel regression models) in Stata 14 (StataCorp, College Station, TX, USA), testing for effects of group (ADHD persisters vs remitters vs controls), time window (pre-stimulus vs post-stimulus), response (correct vs incorrect), and their interaction (group-by-window-by-response). When the three-way interaction was not statistically significant, only statistically significant main effects and two-way interactions were included. For all measures, the within-group degree of change from pre-stimulus to post-stimulus was compared across groups using difference scores to examine how functional connectivity changes with task demands. All models controlled for age and took into account the degree of clustering due to family status. Cohen's $d$ effect sizes are presented along with test statistics, where $d \geq 0.20$ is a small effect, $d \geq 0.50$ a medium effect, and $d \geq 0.80$ a large effect ${ }^{97}$. Given the large number of hypotheses tested, sensitivity analyses applied multipletesting corrections with false discovery rate (FDR) on post hoc tests with the "multproc" package, using the Simes method, which identifies those tests that remain significant ${ }^{98}$.

Since $80 \%$ of our sample consisted of males but groups were not fully matched on sex (Table 1), analyses were performed on the whole sample and then repeated with females (15 ADHD persisters, 41 controls) removed. As in this sample ADHD persisters had a lower IQ than remitters $^{60}$ and childhood IQ predicted ADHD outcome at follow-up ${ }^{71}$, all analyses were also re-run controlling for IQ to examine whether IQ contributes to the results. Finally, even though EEG functional connectivity does not provide a precise localization of functional networks, we examined brain connectivity within and between groups of electrodes from different cortical regions, following previous connectivity studies ${ }^{99,100}$ : analyses were repeated using iCoh values within and between clusters of electrodes in different scalp regions (anterior/central/posterior) and between the two hemispheres (left/right) (Supplementary Fig. 3) (for further details, see Supplementary Material).

\section{Dimensional analysis with ADHD symptoms/impairment}

The association between connectivity metrics and the continua of ADHD symptoms and impairment within individuals with childhood ADHD was examined with random-intercept linear models using DIVA ADHD symptom and impairment scores as independent variables, controlling for age and sex and clustering for family status. Analyses were carried out using standardized scores, thus the beta coefficients are standardized effect sizes comparable to Cohen's $d$. All analyses were re-run, first, correcting for multiple testing, and, second, controlling for IQ.

\section{Association between functional connectivity and cognitive performance}

In an additional analysis, we examined the behavioral significance of the EEG connectivity results in relation to task performance. We tested whether functional connectivity measured by mean iCoh was associated with cognitive performance during the incongruent (highconflict) condition (the same task condition in which connectivity was measured). We previously reported significantly increased mean reaction time (MRT), RTV, and number of errors in ADHD persisters compared to controls in the incongruent condition of this task and intermediate scores with non-significant differences in remitters ${ }^{61}$. The current analyses were restricted to mean iCoh in the pre-stimulus window of correct trials, where differences between ADHD groups and controls were maximal based on categorical analyses. Random-intercept 
linear models on standardized scores tested the association of mean iCoh in theta, alpha, and beta bands as independent variables with MRT, RTV, and the number of errors as dependent variables. These models were run separately in individuals with childhood ADHD and controls, controlling for age and sex and clustering for family status.

\section{Results}

\section{Differences between ADHD persisters, remitters, and controls}

In trials where participants responded correctly, ADHD persisters showed greater clustering coefficient, global efficiency, and mean iCoh and lower path length and diameter compared to controls at all frequency bands in the prestimulus window (before target onset) and only in beta in the post-stimulus windows (Table 2, Fig. 1, Supplementary Fig. 2). Similarly, ADHD remitters showed lower prestimulus diameter in theta and beta, lower pre-stimulus path length in alpha and beta, and lower post-stimulus diameter in beta, compared to controls. ADHD remitters did not differ from persisters in any connectivity measure in correctly-responded trials, except diameter in beta (where remitters were intermediate between controls and persisters and significantly differed from both groups; Table 2). These findings indicate increased connectivity in both ADHD persisters and remitters compared to controls during correct trials. In trials where an error was made, group differences only emerged for clustering coefficient, global efficiency, and mean iCoh in post-stimulus theta: both ADHD persisters and remitters showed reduced values in these measures (indicating lower connectivity) compared to controls but did not differ from each other (Table 2). All three groups showed increased connectivity (greater clustering coefficient, global efficiency, and mean iCoh; decreased path length and diameter) in trials where an incorrect response occurred, compared to trials with correct responses, in both prestimulus and post-stimulus windows (Supplementary Tables 1 and 2), indicating hyper-connectivity before and during incorrect responses. All main and interaction effects are shown in Supplementary Table 2.

Among measures showing significant group-by-window interactions (all in theta, all except diameter in alpha, none in beta; Supplementary Table 2), significant withingroup differences, indicating a change in functional connectivity between the pre-stimulus and the post-stimulus windows, emerged in all groups for all theta connectivity measures; in controls only for clustering coefficient, path length, and mean iCoh in the alpha band; and in both ADHD groups for global efficiency in alpha (Table 3). ADHD persisters and remitters exhibited a significantly lower degree of change compared to controls in all measures of theta connectivity, but no differences emerged between the two ADHD groups (Table 3).
Multiple-testing corrections (controlling the FDR at 15\%) on post hoc group comparisons (separately for ADHD persisters vs controls, ADHD remitters vs controls, ADHD persisters vs remitters) showed that all statistically significant differences between controls and ADHD remitters, and between controls and ADHD persisters remained significant. The only significant difference between ADHD persisters and remitters (in beta diameter) was no longer significant when correcting for multiple testing. All significant group differences on measures of pre-stimulus/ post-stimulus change remained significant after multipletesting corrections.

All results remained unchanged when rerunning analyses on the male-only sample (Supplementary Tables 3 and 4), except that the $p$ values of certain tests that were statistically significant in the full sample became trendlevel effects $(p=0.05-0.10)$. All effect sizes were similar to those on the full sample, suggesting that these nonsignificant results may be due to lower power in this smaller sample.

Results of group comparisons on connectivity measures in pre- and post-stimulus were largely unchanged when IQ was included as a covariate in categorical analyses (Supplementary Table 5). A few differences between persisters and controls on measures of pre-stimulus/poststimulus change in theta and alpha connectivity during error trials were no longer significant (Supplementary Table 6).

Results of analyses on group differences in local connectivity within and between cortical regions were consistent with those on whole-brain connectivity, indicating that functional connectivity profiles were not driven by stronger connectivity within or between particular regions. Specifically, group comparisons showed the same pattern of results when considering functional connectivity within more localized cortical regions (within anterior, central, and posterior regions and within left and right hemispheres), between antero-central, centro-posterior, and antero-posterior regions, and between the two hemispheres (for full results, see Supplementary Material).

\section{Association with ADHD symptoms and impairment}

In dimensional analyses on participants with childhood ADHD, no association emerged between ADHD symptoms and any connectivity measure in theta, alpha, or beta frequencies in correct or error trials (Table 4). Functional impairment was not associated with any connectivity measure in the theta band but showed associations with a subgroup of measures in alpha and beta in correct and error trials (Table 4). Results remained largely unchanged when controlling for IQ (Supplementary Table 7). Statistically significant associations that emerged with ADHD impairment were no longer significant after applying multiple-testing corrections. 
Table 2 Group comparisons on graph-theory and imaginary coherence measures

\begin{tabular}{|c|c|c|c|c|c|c|c|c|}
\hline & & \multicolumn{7}{|c|}{ Group comparison } \\
\hline & & \multirow{2}{*}{$\begin{array}{l}\text { Overall group } \\
p\end{array}$} & \multicolumn{2}{|c|}{ Ctrl vs ADHD-P } & \multicolumn{2}{|c|}{ Ctrl vs ADHD-R } & \multicolumn{2}{|c|}{$\begin{array}{l}\text { ADHD-R vs } \\
\text { ADHD-P }\end{array}$} \\
\hline & & & $p$ & $d$ & $p$ & $d$ & $p$ & $d$ \\
\hline \multicolumn{9}{|l|}{ Theta } \\
\hline \multirow[t]{4}{*}{ Average clustering coefficient } & Pre, Corr & $0.016^{*}$ & $0.004^{* *}$ & 0.63 & 0.880 & 0.29 & 0.139 & 0.35 \\
\hline & Pre, Err & 0.544 & - & - & - & - & - & - \\
\hline & Post, Corr & 0.401 & - & - & - & - & - & - \\
\hline & Post, Err & $<0.001^{* * *}$ & $<0.001^{* * *}$ & 0.35 & $0.017^{*}$ & 0.30 & 0.955 & 0.05 \\
\hline \multirow[t]{4}{*}{ Global efficiency } & Pre, Corr & 0.053 & $0.019^{*}$ & 0.51 & 0.901 & 0.16 & 0.145 & 0.37 \\
\hline & Pre, Err & 0.568 & - & - & - & - & - & - \\
\hline & Post, Corr & 0.189 & - & - & - & - & - & - \\
\hline & Post, Err & $<0.001^{* * *}$ & $<0.001^{* * *}$ & 0.35 & $0.019^{*}$ & 0.30 & 0.916 & 0.05 \\
\hline \multirow[t]{4}{*}{ Path length } & Pre, Corr & $0.012^{*}$ & $<0.001^{* * *}$ & 0.58 & 0.095 & 0.30 & 0.130 & 0.30 \\
\hline & Pre, Err & 0.434 & - & - & - & - & - & - \\
\hline & Post, Corr & 0.338 & - & - & - & - & - & - \\
\hline & Post, Err & 0.122 & - & - & - & - & - & - \\
\hline \multirow[t]{4}{*}{ Diameter } & Pre, Corr & $<0.001^{* * *}$ & $<0.001^{* * *}$ & 0.64 & $0.012^{*}$ & 0.49 & 0.352 & 0.17 \\
\hline & Pre, Err & 0.646 & - & - & - & - & - & - \\
\hline & Post, Corr & 0.976 & - & - & - & - & - & - \\
\hline & Post, Err & 0.279 & - & - & - & - & - & - \\
\hline \multirow[t]{4}{*}{ Mean imaginary coherence } & Pre, Corr & $0.024^{*}$ & $0.007^{* *}$ & 0.60 & 0.952 & -0.25 & 0.140 & 0.35 \\
\hline & Pre, Err & 0.562 & - & - & - & - & - & - \\
\hline & Post, Corr & 0.319 & - & - & - & - & - & - \\
\hline & Post, Err & $<0.001^{* * *}$ & $<0.001^{* * *}$ & 0.35 & $0.019^{*}$ & 0.30 & 0.955 & 0.06 \\
\hline \multicolumn{9}{|l|}{ Alpha } \\
\hline \multirow[t]{4}{*}{ Average clustering coefficient } & Pre, Corr & $0.001^{* *}$ & $<0.001^{* * *}$ & 0.44 & 0.097 & 0.42 & 0.636 & 0.06 \\
\hline & Pre, Err & 0.415 & - & - & - & - & - & - \\
\hline & Post, Corr & 0.328 & - & - & - & - & - & - \\
\hline & Post, Err & 0.084 & - & - & - & - & - & - \\
\hline \multirow[t]{4}{*}{ Global efficiency } & Pre, Corr & $0.003^{* *}$ & $0.002^{* *}$ & 0.32 & 0.054 & 0.39 & 0.976 & 0.04 \\
\hline & Pre, Err & 0.325 & - & - & - & - & - & - \\
\hline & Post, Corr & 0.816 & - & - & - & - & - & - \\
\hline & Post, Err & 0.152 & - & - & - & - & - & - \\
\hline \multirow[t]{4}{*}{ Path length } & Pre, Corr & $<0.001^{* * *}$ & $<0.001^{* * *}$ & 0.32 & $0.005^{* *}$ & 0.47 & 0.539 & 0.13 \\
\hline & Pre, Err & 0.709 & - & - & - & - & - & - \\
\hline & Post, Corr & 0.201 & - & - & - & - & - & - \\
\hline & Post, Err & 0.235 & - & - & - & - & - & - \\
\hline \multirow[t]{2}{*}{ Diameter } & Corr & $<0.001^{* * *}$ & $<0.001^{* * *}$ & 0.41 & 0.054 & 0.30 & 0.610 & 0.13 \\
\hline & Err & 0.444 & - & - & - & - & - & - \\
\hline
\end{tabular}


Table 2 continued

\begin{tabular}{|c|c|c|c|c|c|c|c|c|}
\hline & & \multicolumn{7}{|c|}{ Group comparison } \\
\hline & & \multirow{2}{*}{$\begin{array}{l}\text { Overall group } \\
p\end{array}$} & \multicolumn{2}{|c|}{ Ctrl vs ADHD-P } & \multicolumn{2}{|c|}{ Ctrl vs ADHD-R } & \multicolumn{2}{|c|}{$\begin{array}{l}\text { ADHD-R vs } \\
\text { ADHD-P }\end{array}$} \\
\hline & & & $p$ & $d$ & $p$ & $d$ & $p$ & $d$ \\
\hline \multirow[t]{4}{*}{ Mean imaginary coherence } & Pre, Corr & $0.001^{* *}$ & $<0.001^{* * *}$ & 0.40 & 0.073 & 0.39 & 0.684 & 0.04 \\
\hline & Pre, Err & 0.341 & - & - & - & - & - & - \\
\hline & Post, Corr & 0.501 & - & - & - & - & - & - \\
\hline & Post, Err & 0.064 & - & - & - & - & - & - \\
\hline \multicolumn{9}{|l|}{ Beta } \\
\hline \multirow[t]{2}{*}{ Average clustering coefficient } & Corr & $<0.001^{* * *}$ & $<0.001^{* * *}$ & 0.79 & 0.097 & 0.51 & 0.101 & 0.31 \\
\hline & Err & 0.135 & - & - & - & - & - & - \\
\hline \multirow[t]{2}{*}{ Global efficiency } & Corr & $<0.001^{* * *}$ & $<0.001^{* * *}$ & 0.73 & 0.137 & 0.44 & 0.098 & 0.31 \\
\hline & Err & 0.154 & - & - & - & - & - & - \\
\hline \multirow[t]{2}{*}{ Path length } & Corr & $<0.001^{* * *}$ & $<0.001^{* * *}$ & 0.76 & $0.004^{* *}$ & 0.52 & 0.090 & 0.27 \\
\hline & Err & 0.343 & - & - & - & - & - & - \\
\hline \multirow[t]{2}{*}{ Diameter } & Corr & $<0.001^{* * *}$ & $<0.001^{* * *}$ & 0.83 & $0.003^{* *}$ & 0.53 & $0.044^{*}$ & 0.31 \\
\hline & Err & 0.221 & - & - & - & - & - & - \\
\hline \multirow[t]{2}{*}{ Mean imaginary coherence } & Corr & $<0.001^{* * *}$ & $<0.001^{* * *}$ & 0.77 & 0.097 & 0.49 & 0.101 & 0.31 \\
\hline & Err & 0.135 & - & - & - & - & - & - \\
\hline
\end{tabular}

Notes: Random-intercept linear models tested for main effects of group (ADHD remitters vs ADHD persisters vs controls), time window (pre-stimulus vs post-stimulus) and response (correctly- vs incorrectly-responded trials), two-way interactions (group-by-window, group-by-response, time window-by-response), and three-way interactions (group-by-window-by-response) on connectivity measures. Full results are presented in Supplementary Table 2 . Since neither diameter in the alpha band nor any measures in the beta band showed a significant group-by-window interaction, post hoc effects of group were tested for with correctly- and incorrectlyresponded trials collapsed across pre-stimulus and post-stimulus time windows. Post hoc comparisons between groups were run only on measures showing a significant overall group effect. Age was also included as a covariate of no interest in all analyses. Data in correctly-responded trials were available for 83 ADHD persisters, 22 remitters, and 166 controls and in incorrectly-responded trials for 75 ADHD persisters, 20 remitters, and 145 controls. $d \geq 0.20=$ small effect size, $d \geq 0.50$ $=$ medium effect (in italics), and $d \geq 0.80=$ large effect size (in bold)

$A D H D$ attention-deficit hyperactivity disorder, ADHD-P ADHD persisters, ADHD-R ADHD remitters, Corr trials with correct responses, Ctrl Control group, $d$ Cohen's $d$ effect size, Err trials with incorrect responses, $p$ random-intercept linear model significance testing, Pre pre-stimulus time window, Post post-stimulus time window ${ }^{*} p<0.05 ;{ }^{* *} p<0.01 ;{ }^{* * *} p<0.001$

\section{Association with cognitive performance}

Increased functional connectivity was associated with worse cognitive performance. Specifically, greater mean iCoh in the theta and beta bands showed statistically significant effects on greater RTV in the childhood ADHD group and on greater number of errors in both the childhood ADHD and control groups (Supplementary Table 8). Alpha mean iCoh was not significantly associated with any performance measure in either group. None of the pre-stimulus iCoh connectivity measures had a significant effect on MRT in either group.

\section{Discussion}

Using a network-based EEG functional connectivity approach during an arrow flanker task, our results show widespread hyper-connectivity underlying cognitive control processes, as well as reduced adjustments of connectivity with changed task demands, in individuals with persistent ADHD compared to neurotypical controls. ADHD remitters showed connectivity impairments similar to persisters and differed from controls in most measures of connectivity and of connectivity adjustments. These findings indicate that hyper-connectivity and reduced ability to modulate connectivity patterns with task demands characterize adolescents and young adults with both persistent and remitted ADHD. Atypical functional connectivity during cognitive control processes may thus represent an enduring deficit in adolescents and adults with childhood ADHD, irrespective of their diagnostic outcome.

Two main connectivity impairments emerged in individuals with persistent ADHD compared to controls. First, ADHD persisters showed increased global connectivity (higher iCoh), network clustering (higher clustering coefficient), efficiency (higher global efficiency), and integration (lower path length and diameter) at all 


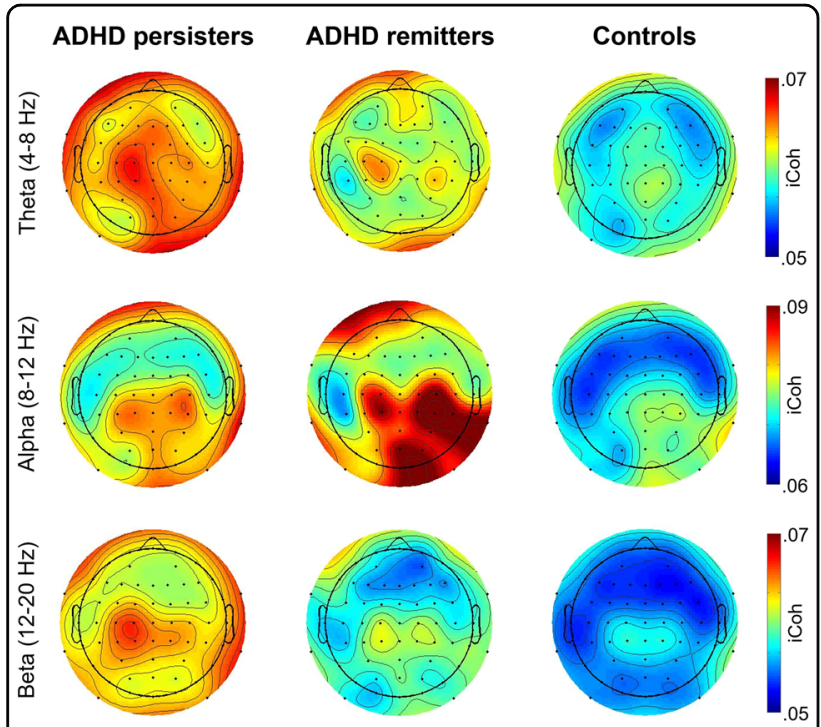

Fig. 1 Topographic maps showing the scalp distribution of the imaginary part of coherence (iCoh) in pre-stimulus theta, alpha, and beta frequencies for correctly-responded trials, divided by group (ADHD persisters, remitters, and controls). We calculated the average between iCoh values for each electrode with all other electrodes, which resulted in one average iCoh value for each channel in each participant. By averaging these values across participants within each group, we obtained distribution maps of average connectivity strength between each scalp site and the rest of the scalp for the three groups. The color scale thus represents the average connectivity of each electrode with all other electrodes (higher in red regions, lower in blue regions)

frequency bands prior to target onset in trials with correct behavioral responses, as well as during target processing in beta oscillations. This increased task-based functional connectivity is consistent with a previous EEG study reporting pre-target over-connectivity in children with $\mathrm{ADHD}^{37}$. More generally, these findings align with previous EEG and fMRI evidence indicating hyperconnectivity in individuals with ADHD during task performance ${ }^{30,33,49}$, but not with other studies, mainly with fMRI, showing task-based hypo-connectivity ${ }^{30-32}$. Some of these inconsistencies may arise from methodological differences between fMRI and EEG, which provide complementary pictures of functional connectivity, especially during cognitive tasks: the former on slower oscillations ${ }^{35}$ and the latter on faster rhythms. Our results suggest that ADHD persisters exhibited hyper-connectivity in theta, alpha, and beta oscillations prior to the onset of incongruent stimuli in this cognitive control task, as well as in beta specifically during target processing. Connectivity in these oscillations during cognitive tasks has been associated with cognitive processes engaging control networks and requiring coordination of activity between distributed and large-scale brain networks ${ }^{4,38,39}$. Here hyperconnectivity in these oscillations in persistent ADHD may reflect exaggerated interactions between brain regions, both during the inactive pre-stimulus period and during cognitive target processing. Considering the high cognitive demands induced by incongruent stimuli in this highly effortful task, which requires a response at every trial, these findings may reflect hyper-connectivity in distributed brain networks underlying higher-level cognitive functions. Second, while all groups showed a significant increase in theta connectivity in changing from pre-stimulus to post-stimulus windows following onset of incongruent stimuli, this change was reduced in ADHD persisters compared to controls. This result in individuals with ADHD may point to a reduced ability to modulate brain connectivity patterns in slow oscillations from a relatively inactive context to a condition requiring cognitive control and conflict monitoring. This finding is in line with previous reports indicating reduced regulation of brain activity in ADHD between different cognitive states $^{101-103}$. Overall, these findings show widespread connectivity impairments underlying cognitive control processes in ADHD persisters and advance our understanding of the neural underpinnings of persistent ADHD in adolescence and early adulthood.

Our study represents the first investigation into EEG connectivity in adolescents and adults with remitted ADHD. In several functional connectivity measures sensitive to impairments in persisters, ADHD remitters were impaired compared to controls and indistinguishable from persisters, consistent with our hypotheses. ADHD remitters also showed the same reduction in all measures of pre-stimulus/post-stimulus change in theta connectivity displayed by persisters. As such, brain connectivity impairments during this cognitive control task were insensitive to ADHD remission/persistence in adolescence and early adulthood and may represent enduring deficits irrespective of current diagnostic status. Findings from dimensional analyses on ADHD severity supported these results, as most connectivity measures in participants with childhood ADHD were unrelated to continuous levels of ADHD symptoms and impairments. Of note, while results of categorical analyses were largely unchanged after correcting for multiple testing, the few significant associations between connectivity and functional impairment (all with small effect sizes) did not survive multiple-testing corrections. These connectivity findings are consistent with previous cognitive-EEG studies, including our previous analyses on this sample ${ }^{60,61}$, reporting that executive-functioning measures are insensitive to ADHD outcomes in adolescence and adulthood $^{60,61,63-65}$. They further extend our earlier findings of no differences between ADHD remitters and persisters on the N2 (reflecting conflict monitoring) and indices of cognitive performance in the same incongruent condition $^{61}$. More broadly, our findings support the co-existence of 
Table 3 Within- and between-group effects on measures of change between pre-stimulus and post-stimulus windows in graph-theory and imaginary coherence measures

\begin{tabular}{|c|c|c|c|c|c|c|c|c|c|c|}
\hline & & \multicolumn{3}{|c|}{ Within-group change } & \multicolumn{6}{|c|}{ Between-group change } \\
\hline & & \multirow{2}{*}{$\begin{array}{l}\text { Ctrl } \\
p\end{array}$} & \multirow{2}{*}{$\begin{array}{l}\text { ADHD-P } \\
p\end{array}$} & \multirow{2}{*}{$\begin{array}{l}\text { ADHD-R } \\
p\end{array}$} & \multicolumn{2}{|c|}{ Ctrl vs ADHD-P } & \multicolumn{2}{|c|}{$\begin{array}{l}\text { Ctrl vs ADHD- } \\
\mathrm{R}\end{array}$} & \multicolumn{2}{|c|}{$\begin{array}{l}\text { ADHD-R vs } \\
\text { ADHD-P }\end{array}$} \\
\hline & & & & & $p$ & $d$ & $p$ & $d$ & $p$ & $d$ \\
\hline \multicolumn{11}{|l|}{ Theta } \\
\hline \multirow[t]{2}{*}{ Average clustering coefficient } & Corr & $<0.001^{* * *}$ & $<0.001^{* * *}$ & $<0.001^{* * *}$ & $0.001^{* *}$ & 0.42 & $0.010^{*}$ & 0.41 & 0.981 & 0.05 \\
\hline & Err & $<0.001^{* * *}$ & $<0.001^{* * *}$ & $<0.001^{* * *}$ & $0.011^{*}$ & 0.33 & 0.618 & 0.06 & 0.370 & 0.26 \\
\hline \multirow[t]{2}{*}{ Global efficiency } & Corr & $<0.001^{* * *}$ & $<0.001^{* * *}$ & $<0.001^{* * *}$ & $0.002^{* *}$ & 0.40 & $0.014^{*}$ & 0.38 & 0.997 & 0.04 \\
\hline & Err & $<0.001^{* * *}$ & $<0.001^{* * *}$ & $<0.001^{* * *}$ & $0.017^{*}$ & 0.31 & 0.643 & 0.06 & 0.400 & 0.25 \\
\hline \multirow[t]{2}{*}{ Path length } & Corr & $<0.001^{* * *}$ & $<0.001^{* * *}$ & $<0.001^{* * *}$ & $<0.001^{* * *}$ & 0.61 & $0.014^{*}$ & 0.44 & 0.506 & 0.19 \\
\hline & Err & $<0.001^{* * *}$ & $<0.001^{* * *}$ & $<0.001^{* * *}$ & 0.058 & 0.27 & 0.776 & 0.11 & 0.209 & 0.36 \\
\hline \multirow[t]{2}{*}{ Diameter } & Corr & $<0.001^{* * *}$ & $<0.001^{* * *}$ & $<0.001^{* * *}$ & $<0.001^{* * *}$ & 0.61 & $0.016^{*}$ & 0.43 & 0.499 & 0.19 \\
\hline & Err & $<0.001^{* * *}$ & $<0.001^{* * *}$ & $<0.001^{* * *}$ & 0.058 & 0.20 & 0.776 & 0.14 & 0.209 & 0.33 \\
\hline \multirow[t]{2}{*}{ Mean imaginary coherence } & Corr & $<0.001^{* * *}$ & $<0.001^{* * *}$ & $<0.001^{* * *}$ & $0.001^{* *}$ & 0.42 & $0.011^{*}$ & 0.40 & 0.995 & 0.05 \\
\hline & Err & $<0.001^{* * *}$ & $<0.001^{* * *}$ & $<0.001^{* * *}$ & $0.013^{*}$ & 0.33 & 0.632 & 0.06 & 0.378 & 0.26 \\
\hline \multicolumn{11}{|l|}{ Alpha } \\
\hline \multirow[t]{2}{*}{ Average clustering coefficient } & Corr & $0.002^{* *}$ & 0.910 & 0.767 & 0.055 & 0.27 & 0.091 & 0.38 & 0.704 & 0.09 \\
\hline & Err & $0.001^{* *}$ & 0.981 & 0.599 & 0.069 & 0.28 & 0.267 & 0.16 & 0.468 & 0.13 \\
\hline \multirow[t]{2}{*}{ Global efficiency } & Corr & 0.728 & $0.004^{* *}$ & $0.045^{*}$ & 0.071 & 0.27 & 0.147 & 0.40 & 0.705 & 0.11 \\
\hline & Err & 0.155 & $0.029^{*}$ & 0.683 & $0.019^{*}$ & 0.38 & 0.140 & 0.25 & 0.389 & 0.15 \\
\hline \multirow[t]{2}{*}{ Path length } & Corr & $0.002^{* *}$ & 0.856 & 0.319 & 0.124 & 0.20 & $0.049^{*}$ & 0.42 & 0.349 & 0.23 \\
\hline & Err & $0.011^{*}$ & 0.831 & 0.931 & $0.023^{*}$ & 0.37 & 0.094 & 0.33 & 0.743 & 0.07 \\
\hline \multirow[t]{2}{*}{ Mean imaginary coherence } & Corr & $0.020^{*}$ & 0.491 & 0.472 & 0.064 & 0.27 & 0.111 & 0.37 & 0.735 & 0.08 \\
\hline & Err & $0.001^{* *}$ & 0.545 & 0.791 & $0.015^{*}$ & 0.40 & 0.087 & 0.30 & 0.469 & 0.13 \\
\hline
\end{tabular}

Notes: Random-intercept linear models tested for main effects of group (ADHD remitters vs ADHD persisters vs controls), time window (pre-stimulus vs post-stimulus) and response (correctly- vs incorrectly-responded trials), two-way interactions (group-by-window, group-by-response, time window-by-response), and three-way interactions (group-by-window-by-response) on connectivity measures. Full results are presented in Supplementary Table 2. Post hoc tests on within- and betweengroup effects of change were run only on measures showing a significant group-by-window interaction. Since this interaction was not significant in diameter in the alpha band or in any measures in the beta band, post-hoc within- and between-groups effects of change were not tested. Age was also included as a covariate of no interest in all analyses. Data in correctly-responded trials were available for 83 ADHD persisters, 22 remitters, and 166 controls; and in incorrectly-responded trials for $75 \mathrm{ADHD}$ persisters, 20 remitters, and 145 controls. $d \geq 0.20=$ small effect size, $d \geq 0.50=$ medium effect (in italics), $d \geq 0.80=$ large effect

$A D H D$ attention-deficit hyperactivity disorder, ADHD-P ADHD persisters, ADHD-R ADHD remitters, Corr trials with correct responses, Ctrl Control group, $d$ Cohen's $d$ effect size, Err trials with incorrect responses, $p$ random-intercept linear model significance testing

${ }^{*} p<0.05 ;{ }^{* *} p<0.01 ;{ }^{* * *} p<0.001$

separate neurobiological processes underpinning developmental pathways to remission and persistence of $\mathrm{ADHD}^{57}$ : functional connectivity during high-conflict trials in this cognitive control task appears unrelated to clinical outcome, unlike measures of less-effortful, non-executive processes (e.g., preparation-vigilance) identified as markers of remission in our previous cognitive-EEG stu$\operatorname{dies}^{60,61,67}$ and in fMRI studies ${ }^{68,104}$. A clinical implication is that connectivity impairments underlying executive-control processes may not be suitable targets for interventions or objective indicators of treatment monitoring, consistent with previous evidence of no effects of stimulants on EEG connectivity in $\mathrm{ADHD}^{47,105}$. Future studies should examine whether EEG functional connectivity during less effortful activities, such as attentional processes, represent markers of remission and candidate targets for new treatments, similar to measures of non-executive processes in our previous studies $^{60,61,67}$.

Of note, while widespread group differences emerged in correctly-responded trials, where participants successfully overcame the conflict generated by incongruent target and flanking stimuli, group differences in error trials emerged only in three measures of post-stimulus theta connectivity. An incorrect response likely represents a 
Table 4 Dimensional associations of graph-theory and imaginary coherence measures with interview-based DIVA ADHD symptom counts and clinical impairment within the ADHD group only $(n=110)$, controlling for age and gender

\begin{tabular}{|c|c|c|c|c|c|}
\hline & & \multicolumn{2}{|l|}{ ADHD symptoms } & \multicolumn{2}{|l|}{ Impairment } \\
\hline & & $\beta(95 \% \mathrm{Cls})$ & $p$ & $\beta(95 \% \mathrm{Cls})$ & $p$ \\
\hline \multicolumn{6}{|l|}{ Theta } \\
\hline \multirow[t]{4}{*}{ Average clustering coefficient } & Pre, Corr & $-0.005(-0.202 ; 0.193)$ & 0.964 & $0.160(-0.065 ; 0.384)$ & 0.163 \\
\hline & Pre, Err & $0.020(-0.177 ; 0.216)$ & 0.844 & $0.178(-0.040 ; 0.398)$ & 0.110 \\
\hline & Post, Corr & $-0.021(-0.218 ; 0.174)$ & 0.827 & $0.176(-0.0041 ; 0.393)$ & 0.111 \\
\hline & Post, Err & $-0.088(-0.259 ; 0.084)$ & 0.315 & $-0.068(-0.263 ; 0.127)$ & 0.494 \\
\hline \multirow[t]{4}{*}{ Global efficiency } & Pre, Corr & $-0.037(-0.216 ; 0.142)$ & 0.685 & $0.089(-0.115 ; 0.292)$ & 0.393 \\
\hline & Pre, Err & $0.004(-0.181 ; 0.189)$ & 0.969 & $0.167(-0.280 ; 0.373)$ & 0.110 \\
\hline & Post, Corr & $-0.043(-0.267 ; 0.152)$ & 0.667 & $0.145(-0.074 ; 0.365)$ & 0.194 \\
\hline & Post, Err & $-0.111(-0.279 ; 0.057)$ & 0.196 & $-0.152(-0.344 ; 0.040)$ & 0.120 \\
\hline \multirow[t]{4}{*}{ Path length } & Pre, Corr & $0.033(-0.145 ; 0.211)$ & 0.716 & $-0.123(-0.322 ; 0.076)$ & 0.226 \\
\hline & Pre, Err & $-0.027(-0.231 ; 0.178)$ & 0.797 & $-0.175(-0.402 ; 0.053)$ & 0.132 \\
\hline & Post, Corr & $0.067(-0.140 ; 0.273)$ & 0.528 & $-0.100(-0.332 ; 0.131)$ & 0.395 \\
\hline & Post, Err & $0.108(-0.092 ; 0.308)$ & 0.290 & $0.056(-0.171 ; 0.282)$ & 0.630 \\
\hline \multirow[t]{4}{*}{ Diameter } & Pre, Corr & $0.049(-0.135 ; 0.232)$ & 0.601 & $-0.109(-0.321 ; 0.102)$ & 0.310 \\
\hline & Pre, Err & $-0.043(-0.251 ; 0.165)$ & 0.685 & $-0.168(-0.107 ; 0.028)$ & 0.153 \\
\hline & Post, Corr & $0.030(-0.161 ; 0.222)$ & 0.756 & $-0.116(-0.329 ; 0.098)$ & 0.287 \\
\hline & Post, Err & $0.100(-0.100 ; 0.300)$ & 0.328 & $0.020(-0.202 ; 0.242)$ & 0.861 \\
\hline \multirow[t]{4}{*}{ Mean imaginary coherence } & Pre, Corr & $0.013(-0.205 ; 0.180)$ & 0.898 & $0.142(-0.077 ; 0.361)$ & 0.204 \\
\hline & Pre, Err & $0.015(-0.178 ; 0.208)$ & 0.878 & $0.175(-0.040 ; 0.390)$ & 0.110 \\
\hline & Post, Corr & $-0.028(-0.224 ; 0.168)$ & 0.778 & $0.167(-0.051 ; 0.385)$ & 0.134 \\
\hline & Post, Err & $-0.096(-0.266 ; 0.074)$ & 0.268 & $-0.101(-0.295 ; 0.093)$ & 0.306 \\
\hline \multicolumn{6}{|l|}{ Alpha } \\
\hline \multirow[t]{4}{*}{ Average clustering coefficient } & Pre, Corr & $0.014(-0.186 ; 0.215)$ & 0.894 & $0.044(-0.186 ; 0.274)$ & 0.708 \\
\hline & Pre, Err & $0.051(-0.130 ; 0.233)$ & 0.578 & $0.157(-0.049 ; 0.363)$ & 0.135 \\
\hline & Post, Corr & $0.064(-0.121 ; 0.249)$ & 0.500 & $0.256(0.056 ; 0.456)$ & $0.012^{*}$ \\
\hline & Post, Err & $0.117(-0.063 ; 0.297)$ & 0.204 & $0.223(0.017 ; 0.429)$ & $0.034^{*}$ \\
\hline \multirow[t]{4}{*}{ Global efficiency } & Pre, Corr & $-0.027(-0.231 ; 0.176)$ & 0.794 & $0.091(-0.326 ; 0.145)$ & 0.450 \\
\hline & Pre, Err & $0.033(-0.160 ; 0.226)$ & 0.738 & $0.130(-0.089 ; 0.349)$ & 0.245 \\
\hline & Post, Corr & $0.052(-0.125 ; 0.230)$ & 0.563 & $0.199(0.004 ; 0.394)$ & $0.046^{*}$ \\
\hline & Post, Err & $0.108(-0.063 ; 0.280)$ & 0.216 & $0.222(0.021 ; 0.419)$ & $0.031^{*}$ \\
\hline \multirow[t]{4}{*}{ Path length } & Pre, Corr & $<0.001(-0.184 ; 0.183)$ & 0.998 & $0.080(-0.129 ; 0.289)$ & 0.452 \\
\hline & Pre, Err & $-0.026(-0.219 ; 0.167)$ & 0.793 & $-0.132(-0.347 ; 0.083)$ & 0.229 \\
\hline & Post, Corr & $-0.052(-0.237 ; 0.132)$ & 0.580 & $-0.202(-0.404 ; 0.000)$ & 0.050 \\
\hline & Post, Err & $-0.131(-0.319 ; 0.057)$ & 0.172 & $-0.222(-0.436 ;-0.008)$ & $0.042^{*}$ \\
\hline \multirow[t]{3}{*}{ Diameter } & Pre, Corr & $-0.027(-0.223 ; 0.129)$ & 0.784 & $-0.051(-0.277 ; 0.175)$ & 0.659 \\
\hline & Pre, Err & $-0.080(-0.272 ; 0.113)$ & 0.417 & $-0.177(-0.397 ; 0.043)$ & 0.114 \\
\hline & Post, Corr & $-0.053(-0.245 ; 0.139)$ & 0.588 & $-0.232(-0.442 ;-0.023)$ & $0.030^{*}$ \\
\hline
\end{tabular}


Table 4 continued

\begin{tabular}{|c|c|c|c|c|c|}
\hline & & \multicolumn{2}{|l|}{ ADHD symptoms } & \multicolumn{2}{|l|}{ Impairment } \\
\hline & & $\beta(95 \% \mathrm{Cls})$ & $p$ & $\beta(95 \% \mathrm{Cls})$ & $p$ \\
\hline \multirow{5}{*}{ Mean imaginary coherence } & Post, Err & $-0.134(-0.332 ; 0.064)$ & 0.185 & $-0.201(-0.428 ; 0.027)$ & 0.083 \\
\hline & Pre, Corr & $0.003(-0.202 ; 0.195)$ & 0.973 & $-0.003(-0.229 ; 0.224)$ & 0.981 \\
\hline & Pre, Err & $0.049(-0.152 ; 0.251)$ & 0.631 & $0.165(-0.063 ; 0.393)$ & 0.156 \\
\hline & Post, Corr & $0.062(-0.121 ; 0.245)$ & 0.505 & $0.242(0.045 ; 0.441)$ & $0.016^{*}$ \\
\hline & Post, Err & $0.115(-0.063 ; 0.294)$ & 0.204 & $0.223(0.018 ; 0.427)$ & $0.033^{*}$ \\
\hline \multicolumn{6}{|l|}{ Beta } \\
\hline \multirow[t]{4}{*}{ Average clustering coefficient } & Pre, Corr & $0.124(-0.100 ; 0.349)$ & 0.278 & $0.306(0.062 ; 0.550)$ & $0.014^{*}$ \\
\hline & Pre, Err & $0.051(-0.149 ; 0.252)$ & 0.613 & $0.203(-0.022 ; 0.429)$ & 0.077 \\
\hline & Post, Corr & $0.093(-0.141 ; 0.328)$ & 0.435 & $0.264(0.001 ; 0.527)$ & $0.049 *$ \\
\hline & Post, Err & $0.045(-0.160 ; 0.250)$ & 0.666 & $0.166(-0.062 ; 0.393)$ & 0.153 \\
\hline \multirow[t]{4}{*}{ Global efficiency } & Pre, Corr & $0.100(-0.119 ; 0.319)$ & 0.372 & $0.299(0.061 ; 0.536)$ & $0.014^{*}$ \\
\hline & Pre, Err & $0.047(-0.155 ; 0.248)$ & 0.650 & $0.210(-0.016 ; 0.436)$ & 0.069 \\
\hline & Post, Corr & $0.068(-0.167 ; 0.302)$ & 0.572 & $0.248(-0.015 ; 0.512)$ & 0.065 \\
\hline & Post, Err & $0.042(-0.163 ; 0.248)$ & 0.688 & $0.166(-0.062 ; 0.394)$ & 0.152 \\
\hline \multirow[t]{4}{*}{ Path length } & Pre, Corr & $-0.092(-0.289 ; 0.105)$ & 0.361 & $-0.233(-0.450 ;-0.016)$ & $0.035^{*}$ \\
\hline & Pre, Err & $-0.067(-0.264 ; 0.131)$ & 0.508 & $-0.198(-0.419 ; 0.023)$ & 0.080 \\
\hline & Post, Corr & $-0.073(-0.280 ; 0.134)$ & 0.490 & $-0.191(-0.425 ; 0.043)$ & 0.110 \\
\hline & Post, Err & $-0.080(-0.283 ; 0.124)$ & 0.444 & $-0.163(-0.387 ; 0.061)$ & 0.153 \\
\hline \multirow[t]{4}{*}{ Diameter } & Pre, Corr & $-0.118(-0.318 ; 0.083)$ & 0.251 & $-0.253(-0.474 ;-0.033)$ & $0.024^{*}$ \\
\hline & Pre, Err & $-0.094(-0.301 ; 0.112)$ & 0.372 & $-0.166(-0.395 ; 0.063)$ & 0.157 \\
\hline & Post, Corr & $-0.105(-0.312 ; 0.102)$ & 0.320 & $-0.190(-0.425 ; 0.045)$ & 0.114 \\
\hline & Post, Err & $-0.089(-0.299 ; 0.122)$ & 0.410 & $-0.124(-0.357 ; 0.108)$ & 0.294 \\
\hline \multirow[t]{4}{*}{ Mean imaginary coherence } & Pre, Corr & $0.118(-0.105 ; 0.341)$ & 0.301 & $0.305(0.063 ; 0.548)$ & $0.013^{*}$ \\
\hline & Pre, Err & $0.051(-0.150 ; 0.251)$ & 0.620 & $0.207(-0.019 ; 0.432)$ & 0.072 \\
\hline & Post, Corr & $0.085(-0.150 ; 0.321)$ & 0.478 & $0.259(-0.005 ; 0.523)$ & 0.054 \\
\hline & Post, Err & $0.044(-0.162 ; 0.249)$ & 0.676 & $0.166(-0.062 ; 0.394)$ & 0.153 \\
\hline
\end{tabular}

Notes: Random-intercept linear models tested for the effect of ADHD symptom count/impairment on each connectivity measure. $\beta \geq 0.20=$ small effect size, $\beta \geq 0.50$ $=$ medium effect, $\beta \geq 0.80$ = large effect. Data in correctly-responded trials were available for 105 childhood ADHD participants (83 ADHD persisters, 22 remitters) and in incorrectly-responded trials for 95 childhood ADHD participants (75 ADHD persisters, 20 remitters)

$A D H D$ attention-deficit/hyperactivity disorder, $\beta$ standardized regression coefficient, $C I$ confidence interval, Corr trials with correct responses, DIVA Diagnostic Interview for ADHD in Adults, Err trials with incorrect responses, $p$ random-intercept linear model significance testing, Pre pre-stimulus time window, Post poststimulus time window

${ }^{*} p<0.05$

failure of cognitive control, required for selection of a correct response in the highly challenging incongruent condition. The limited group differences in connectivity during incorrect responses may suggest that a suboptimal pattern of functional connectivity may attenuate the differences in brain-network profiles between neurotypical individuals and individuals with ADHD, who are prone to making more incorrect responses in this task ${ }^{61}$. In addition, in all groups functional connectivity was increased during incorrect responses compared to correct responses, both during the inactive pre-stimulus window and during processing of incongruent stimuli. This is in line with the interpretation that hyper-connectivity displayed in the ADHD groups was dysfunctional during this task. An additional analysis testing whether hyperconnectivity was related to impairments in cognitive performance during this task further confirmed this pattern, as pre-stimulus hyper-connectivity in theta and beta oscillations was associated with fewer correct responses in individuals with childhood ADHD and controls and with 
increased RTV in individuals with childhood ADHD. Increased functional connectivity in both ADHD persisters and remitters may thus contribute to the lack of differences in cognitive performance measures between ADHD remitters and persisters reported in our previous study $^{61}$. Overall, a suboptimal pattern of hyperconnectivity underlying cognitive control processes may lead to dysfunctional behavioral responses, both in neurotypical individuals and in individuals with childhood ADHD.

A limitation of this study is that, despite the large sample, the low ADHD remission rate at follow-up resulted in a relatively small group of remitters. Therefore, we could not exclude the possibility that some nonsignificant group differences could be due to low power. However, the moderate effect sizes $(d=0.38-0.53)$ between ADHD remitters and controls, but negligible or small ( $d=0.02-0.36)$ between remitters and persisters, in measures showing ADHD persister-control differences suggest that we had sufficient power to detect, with the current sample sizes, differences in connectivity with at least moderate effect sizes. In addition, our sample included young adults as well as adolescents who are still undergoing rapid cortical maturation. While analyses controlled for age, future follow-up assessments with participants having reached adulthood could provide further insight into developmental patterns. Finally, the relatively poor spatial resolution of scalp-EEG did not allow precise localization of the brain networks. Yet, the current EEG connectivity analyses allowed precise temporal resolution during two short time windows and both correct and incorrect behavioral responses, as well as connectivity estimates unaffected by volume-conduction artifacts and examination of whole-brain network properties. The results of local connectivity within and between cortical regions were further consistent with those of whole-brain analyses, indicating comparable effects in more localized networks.

In conclusion, we report new evidence of shared atypical task-based connectivity profiles in adolescents and young adults with persistent and remitted ADHD. These connectivity alterations may represent enduring deficits and neural signatures associated with having a history of childhood ADHD, but appear unrelated to follow-up diagnostic status. Connectivity impairments underlying executive processes may represent associated characteristics or risk factors in $\mathrm{ADHD}^{10}$, which do not follow the developmental pathways of clinical profiles. Future studies should explore the presence of potential compensatory mechanisms that may enable developmental improvements in clinical profiles and non-executive cognitive processes in individuals with remitted $\mathrm{ADHD}^{60,61,67}$, despite enduring functional connectivity alterations during cognitive control.

\section{Acknowledgements}

We thank all who made this research possible: our participants; their families; and research workers Jessica Deadman, Hannah Collyer, and Sarah-Jane Gregori. This project was supported by generous grants from Action Medical Research and the Peter Sowerby Charitable Foundation (GN1777) to J.K. Initial cognitive assessments of the ADHD and control groups in childhood and the recruitment of the control sample were supported by UK Medical Research Council grant (G0300189) to J.K. Initial sample recruitment of the ADHD group was supported by NIMH grant (R01MH062873) to S.V.F. G.M. was supported by a $1+3$ PhD studentship awarded by the MRC Social, Genetic and Developmental Psychiatry Centre, Institute of Psychiatry, Psychology and Neuroscience, King's College London (G9817803) and by a Short-term fellowship by the European Molecular Biology Organization (EMBO ASTF 2182015). P.A. is supported by generous grants from the National Institute for Health Research (NIHR) Biomedical Research Centre for Mental Health at King's College London, Institute of Psychiatry, Psychology and Neuroscience and South London and Maudsley National Health Service (NHS) Foundation Trust. I. B. is supported by the NIHR Biomedical Research Centre at South London and Maudsley NHS Foundation Trust and by the NIHR Collaboration for Leadership in Applied Health Research and Care South London at King's College Hospital NHS Foundation Trust. This paper represents independent research partfunded by the NIHR Biomedical Research Centre at South London and Maudsley NHS Foundation Trust and King's College London. The views expressed are those of the authors and not necessarily those of the NHS, the $\mathrm{NIHR}$, or the Department of Health and Social Care. A preprint version of this manuscript was deposited on bioRxiv (https://doi.org/10.1101/201772).

\section{Author details}

${ }^{1}$ Social, Genetic and Developmental Psychiatry Centre, Institute of Psychiatry, Psychology and Neuroscience, King's College London, London, UK. ${ }^{2}$ Department of Psychiatry and Behavioral Health, State University New York (SUNY) Stony Brook University, Stony Brook, NY, USA. ${ }^{3}$ Semel Institute for Neuroscience and Human Behavior, University of California, Los Angeles (UCLA), Los Angeles, CA, USA. ${ }^{4}$ Department of Biostatistics, Institute of Psychiatry, Psychology and Neuroscience, King's College London, London, UK. ${ }^{5}$ HRL Laboratories, Malibu, CA, USA

\section{Compliance with ethical standards}

\section{Conflict of interest}

P.A. has received funding for research by Vifor Pharma and has given sponsored talks and been an advisor for Shire, Janssen-Cilag, Eli-Lilly, Flynn Pharma, and Pfizer regarding the diagnosis and treatment of ADHD. All funds are received by King's College London and used for studies of ADHD. The other authors declare that they have no conflict of interest.

\section{Ethical approval}

All procedures were in accordance with the ethical standards of the responsible committee on human experimentation (London-Surrey Borders Research Ethics Committee) and with the Helsinki Declaration of 1975 (as revised in 1983).

\section{Publisher's note}

Springer Nature remains neutral with regard to jurisdictional claims in published maps and institutional affiliations.

Supplementary Information accompanies this paper at (https://doi.org/ 10.1038/s41398-019-0469-7).

Received: 27 April 2018 Revised: 10 January 2019 Accepted: 23 March 2019 Published online: 12 April 2019

\section{References}

1. Deco, G. \& Kringelbach, M. Metastability and coherence: extending the communication through coherence hypothesis using a whole-brain computational perspective. Trends Neurosci. 39, 432 (2016).

2. Bullmore, E. \& Sporns, O. Complex brain networks: graph theoretical analysis of structural and functional systems. Nat. Rev. Neurosci. 10, 186-198 (2009). 
3. Coben, R., Mohammad-Rezazadeh, I., Frohich, J., Jurgiel, J. \& Michelini, G. Imaging brain connectivity in autism spectrum disorder. In M. F., Casanova, A., El-Baz. \& J. S, Suri (eds). Autism Imaging and Devices Ch. 13. CRC Press: Boca Raton, (2017)

4. Wang, X. J. Neurophysiological and computational principles of cortical rhythms in cognition. Physiol. Rev. 90, 1195-1268 (2010).

5. Castellanos, F. X. \& Aoki, Y. Intrinsic functional connectivity in attentiondeficit/hyperactivity disorder: a science in development. Biol. Psychiatry Cogn. Neurosci. Neuroimaging 1, 253-261 (2016).

6. Kitzbichler, M. G. et al. Altered development and multifaceted band-specific abnormalities of resting state networks in autism. Biol. Psychiatry 77, 794-804 (2015).

7. Xing, M. et al. Resting-state theta band connectivity and graph analysis in generalized social anxiety disorder. Neuroimage Clin. 13, 24-32 (2017).

8. Castellanos, F. X. \& Proal, E. Large-scale brain systems in ADHD: beyond the prefrontal-striatal model. Trends Cogn. Sci. 16, 17-26 (2012).

9. Cortese, S. et al. Toward systems neuroscience of ADHD: a meta-analysis of 55 fMRI studies. Am. J. Psychiatry 169, 1038-1055 (2012).

10. Johnson, M. H. Executive function and developmental disorders: the flip side of the coin. Trends Cogn Sci 16, 454-457 (2012).

11. Halperin, J. M. \& Schulz, K. P. Revisiting the role of the prefrontal cortex in the pathophysiology of attention-deficit/hyperactivity disorder. Psychol. Bull. 132, 560-581 (2006).

12. Konrad, K. \& Eickhoff, S. B. Is the ADHD brain wired differently? A review on structural and functional connectivity in attention deficit hyperactivity disorder. Hum. Brain Mapp. 31, 904-916 (2010)

13. Sergeant, J. A. Modeling attention-deficit/hyperactivity disorder: a critica appraisal of the cognitive-energetic model. Biol. Psychiatry 57, 1248-1255 (2005)

14. Uddin, L. Q. et al. Network homogeneity reveals decreased integrity of default-mode network in ADHD. J. Neurosci. Methods 169, 249-254 (2008).

15. Cao, M., Shu, N., Cao, Q., Wang, Y. \& He, Y. Imaging functional and structura brain connectomics in attention-deficit/hyperactivity disorder. Mol. Neurobiol. 50, 1111-1123 (2014).

16. Sripada, C. et al. Disrupted network architecture of the resting brain in attention-deficit/hyperactivity disorder. Hum. Brain Mapp. 35, 4693-4705 (2014).

17. Fair, D. A. et al. Atypical default network connectivity in youth with attentiondeficit/hyperactivity disorder. Biol. Psychiatry 68, 1084-1091 (2010).

18. Sun, L. et al. Abnormal functional connectivity between the anterior cingulate and the default mode network in drug-naive boys with attention deficit hyperactivity disorder. Psychiatry Res. 201, 120-127 (2012).

19. Castellanos, F. X. et al. Cingulate-precuneus interactions: a new locus of dysfunction in adult attention-deficit/hyperactivity disorder. Biol. Psychiatry 63, 332-337 (2008)

20. Barbera, A. D. J. et al. Connectivity supporting attention in children with attention deficit hyperactivity disorder. Neuroimage Clin. 7, 68-81 (2015).

21. Hoekzema, E. et al. An independent components and functional connectivity analysis of resting state fMRI data points to neural network dysregulation in adult ADHD. Hum. Brain Mapp. 35, 1261-1272 (2014).

22. McCarthy, $\mathrm{H}$. et al. Attention network hypoconnectivity with default and affective network hyperconnectivity in adults diagnosed with attentiondeficit/hyperactivity disorder in childhood. JAMA Psychiatry 70, 1329-1337 (2013).

23. Tian, L. et al. Altered resting-state functional connectivity patterns of anterior cingulate cortex in adolescents with attention deficit hyperactivity disorder. Neurosci. Lett. 400, 39-43 (2006).

24. Sidlauskaite, J., Sonuga-Barke, E., Roeyers, H. \& Wiersema, J. R. Altered intrinsic organisation of brain networks implicated in attentional processes in adult attention-deficit/hyperactivity disorder: a resting-state study of attention, default mode and salience network connectivity. Eur. Arch. Psychiatry Clin. Neurosci. 266, 349-357 (2016).

25. Mostert, J. C. et al. Characterising resting-state functional connectivity in a large sample of adults with ADHD. Prog. Neuropsychopharmacol. Biol. Psychiatry 67, 82-91 (2016).

26. Qian, $X_{\text {., }}$ et al. Large-scale brain functional network topology disruptions underlie symptom heterogeneity in children with attention-deficit/hyperactivity disorder. Neuroimage Clin. 21, 101600 (2019).

27. Ernst, M., Torrisi, S., Balderston, N., Grillon, C. \& Hale, E. A. fMRl functional connectivity applied to adolescent neurodevelopment. Annu. Rev. Clin. Psychol. 11, 361-377 (2015).
28. Finn, E. S. et al. Can brain state be manipulated to emphasize individual differences in functional connectivity? Neuroimage 160, 140-151 (2017).

29. Rubia, K. et al. Methylphenidate normalises activation and functional connectivity deficits in attention and motivation networks in medication-naive children with $\mathrm{ADHD}$ during a rewarded continuous performance task. Neuropharmacology 57, 640-652 (2009).

30. van Rooij, D. et al. Altered neural connectivity during response inhibition in adolescents with attention-deficit/hyperactivity disorder and their unaffected siblings. Neuroimage Clin. 7, 325-335 (2015).

31. Cubillo, A. et al. Reduced activation and inter-regional functional connectivity of fronto-striatal networks in adults with childhood attention-deficit hyperactivity disorder (ADHD) and persisting symptoms during tasks of motor inhibition and cognitive switching. J. Psychiatr. Res. 44, 629-639 (2010).

32. Vloet, T. D. et al. Neural mechanisms of interference control and time discrimination in attention-deficit/hyperactivity disorder. J. Am. Acad. Child Adolesc. Psychiatry 49, 356-367 (2010).

33. $\mathrm{Ma}$, I. et al. Ventral striatal hyperconnectivity during rewarded interference control in adolescents with ADHD. Cortex 82, 225-236 (2016).

34. Friedman, L. A. \& Rapoport, J. L. Brain development in ADHD. Curr. Opin. Neurobiol. 30, 106-111 (2015)

35. Lewis, L. D., Setsompop, K., Rosen, B. R. \& Polimeni, J. R. Fast fMRI can detect oscillatory neural activity in humans. Proc. Natl Acad. Sci. USA 113 E6679-e6685 (2016).

36. Coben, R., Mohammad-Rezazadeh, I. \& Cannon, R. L. Using quantitative and analytic EEG methods in the understanding of connectivity in autism spectrum disorders: a theory of mixed over- and under-connectivity. Front. Hum. Neurosci. 8, 45 (2014).

37. Silberstein, R. B. et al. Brain functional connectivity abnormalities in attentiondeficit hyperactivity disorder. Brain Behav. 6, e00583 (2016).

38. Buzsaki, G. \& Draguhn, A. Neuronal oscillations in cortical networks. Science 304, 1926-1929 (2004).

39. Uhlhaas, P. J. \& Singer, W. Neural synchrony in brain disorders: relevance for cognitive dysfunctions and pathophysiology. Neuron 52, 155-168 (2006).

40. Wig, G. S. Segregated systems of human brain networks. Trends Cogn. Sci. 21, 981-996 (2017).

41. Zavala, B. et al. Human subthalamic nucleus-medial frontal cortex theta phase coherence is involved in conflict and error related cortical monitoring. Neuroimage 137, 178-187 (2016).

42. Almabruk, T., lyer, K., Tan, T., Roberts, G. \& Anderson, M. An EEG coherencebased analysis approach for investigating response conflict processes in 7 and 9-year old children. Conf. Proc. IEEE Eng. Med. Biol. Soc. 2884-2887 (2015)

43. Suzuki, $\mathrm{K}$. et al. The relationship between the superior frontal cortex and alpha oscillation in a flanker task: simultaneous recording of electroencephalogram (EEG) and near infrared spectroscopy (NIRS). Neurosci. Res. 131, 30-35 (2018)

44. Janssens, C., De Loof, E., Boehler, C. N., Pourtois, G. \& Verguts, T. Occipital alpha power reveals fast attentional inhibition of incongruent distractors. Psychophysiology https://doi.org/10.1111/psyp.13011 (2018).

45. Mazaheri, A. et al. Differential oscillatory electroencephalogram between attention-deficit/hyperactivity disorder subtypes and typically developing adolescents. Biol. Psychiatry 76, 422-429 (2014).

46. Clarke, A. R. et al. Coherence in children with attention-deficit/hyperactivity disorder and excess beta activity in their EEG. Clin. Neurophysiol. 118 1472-1479 (2007).

47. Dupuy, F. E., Clarke, A. R., Barry, R. J., McCarthy, R. \& Selikowitz, M. EEG coherence in girls with attention-deficit/hyperactivity disorder: stimulant effects in good responders. Int. J. Psychophysiol. 70, 151-157 (2008).

48. Barry, R. J., Clarke, A. R., McCarthy, R., Selikowitz, M. \& Johnstone, S. J. EEG coherence adjusted for inter-electrode distance in children with attentiondeficit/hyperactivity disorder. Int. J. Psychophysiol. 58, 12-20 (2005).

49. Murias, M., Swanson, J. M. \& Srinivasan, R. Functional connectivity of frontal cortex in healthy and ADHD children reflected in EEG coherence. Cereb. Cortex 17, 1788-1799 (2007).

50. Mazaheri, A. et al. Functional disconnection of frontal cortex and visual cortex in attention-deficit/hyperactivity disorder. Biol. Psychiatry 67, 617-623 (2010).

51. Nolte, G. et al. Identifying true brain interaction from EEG data using the imaginary part of coherency. Clin. Neurophysiol. 115, 2292-2307 (2004).

52. Nunez, P. L. et al. EEG coherency. I: Statistics, reference electrode, volume conduction, Laplacians, cortical imaging, and interpretation at multiple scales. Electroencephalogr. Clin. Neurophysiol. 103, 499-515 (1997). 
53. Rubinov, M. \& Sporns, O. Complex network measures of brain connectivity: uses and interpretations. Neuroimage 52, 1059-1069 (2010).

54. Liu, T., Chen, Y., Lin, P. \& Wang, J. Small-world brain functional networks in children with attention-deficit/hyperactivity disorder revealed by EEG synchrony. Clin. EEG Neurosci. 46, 183-191 (2015).

55. Faraone, S. V., Biederman, J. \& Mick, E. The age-dependent decline of attention deficit hyperactivity disorder: a meta-analysis of follow-up studies. Psychol. Med. 36, 159-165 (2006).

56. Sibley, M. H. et al. Defining ADHD symptom persistence in adulthood: optimizing sensitivity and specificity. J. Child Psychol. Psychiatry 58, 655-662 (2016)

57. Sudre, G., Mangalmurti, A. \& Shaw, P. Growing out of attention deficit hyperactivity disorder: insights from the 'remitted' brain. Neurosci. Biobehav. Rev. 94, 198-209 (2018).

58. Franke, B. et al. Live fast, die young? A review on the developmental trajectories of ADHD across the lifespan. Eur. Neuropsychopharmacol. 28, 1059-1088 (2018)

59. Shaw, P. et al. Trajectories of cerebral cortical development in childhood and adolescence and adult attention-deficit/hyperactivity disorder. Biol. Psychiatry 74, 599-606 (2013)

60. Cheung, C. H. et al. Cognitive and neurophysiological markers of ADHD persistence and remission. Br. J. Psychiatry 208, 548-555 (2016).

61. Michelini, G. et al. Attention-deficit/hyperactivity disorder remission is linked to better neurophysiological error detection and attention-vigilance processes. Biol. Psychiatry 80, 923-932 (2016).

62. Cortese, S. et al. White matter alterations at 33-year follow-up in adults with childhood attention-deficit/hyperactivity disorder. Biol. Psychiatry 74, 591-598 (2013).

63. Pazvantoglu, O. et al. Neuropsychological weaknesses in adult ADHD; cognitive functions as core deficit and roles of them in persistence to adulthood. J. Int. Neuropsychol. Soc. 18, 819-826 (2012).

64. Biederman, J. et al. Are cognitive deficits in attention deficit/hyperactivity disorder related to the course of the disorder? A prospective controlled follow-up study of grown up boys with persistent and remitting course. Psychiatry Res. 170, 177-182 (2009).

65. McAuley, T., Crosbie, J., Charach, A. \& Schachar, R. The persistence of cognitive deficits in remitted and unremitted ADHD: a case for the stateindependence of response inhibition. J. Child Psychol. Psychiatry 55, 292-300 (2014).

66. Roman-Urrestarazu, A. et al. Brain structural deficits and working memory fMRI dysfunction in young adults who were diagnosed with ADHD in adolescence. Eur. Child Adolesc. Psychiatry 25, 529-538 (2016).

67. James, S.-N. et al. Peripheral hypoarousal but not preparation-vigilance impairment endures in ADHD remission. J. Atten. Disord. https://doi.org/ 10.1177/1087054717698813 (2017)

68. Clerkin, S. M. et al. Thalamo-cortical activation and connectivity during response preparation in adults with persistent and remitted ADHD. Am. J. Psychiatry 170, 1011-1019 (2013).

69. Kuntsi, J. et al. Separation of cognitive impairments in attention-deficit/ hyperactivity disorder into 2 familial factors. Arch. Gen. Psychiatry 67 1159-1167 (2010).

70. Michelini, G. et al. The etiological structure of cognitive-neurophysiological impairments in ADHD in adolescence and young adulthood. J. Atten. Disord. https://doi.org/10.1177/1087054718771191 (2018).

71. Cheung, C. H. et al. Childhood predictors of adolescent and young adult outcome in ADHD. J Psychiatr. Res. 62, 92-100 (2015).

72. Wechsler, D. Wechsler Abbreviated Scale of Intelligence (WASI) (Psychological Corporation, San Antonio, TX, 1999).

73. Ramos-Quiroga, J. A. et al. Criteria and concurrent validity of DIVA 2.0: a semistructured diagnostic interview for adult ADHD. J. Atten. Disord. https://doi. org/10.1177/1087054716646451 (2016).

74. Barkley, R. A. \& Murphy, K. Attention Deficit Hyperactivity Disorder: A Clinical Workbook 3rd edn (Guildford Press, New York, 2006).

75. Du Rietz, E. et al. Self-report of ADHD shows limited agreement with objective markers of persistence and remittance. J. Psychiatr. Res. 82, 91-99 (2016).

76. Albrecht, B. et al. Action monitoring in boys with attention-deficit/hyperactivity disorder, their nonaffected siblings, and normal control subjects: evidence for an endophenotype. Biol. Psychiatry 64, 615-625 (2008).
77. McLoughlin, G. et al. Performance monitoring is altered in adult ADHD: a familial event-related potential investigation. Neuropsychologia 47, 3134-3142 (2009).

78. Jung, T. P. et al. Removing electroencephalographic artifacts by blind source separation. Psychophysiology 37, 163-178 (2000).

79. Hinkley, L. B. et al. Cognitive impairments in schizophrenia as assessed through activation and connectivity measures of magnetoencephalography (MEG) data. Front. Hum. Neurosci. 3, 73 (2010).

80. Palva, S. \& Palva, J. M. Discovering oscillatory interaction networks with $M$ / EEG: challenges and breakthroughs. Trends Cogn. Sci. 16, 219-230 (2012).

81. Onton, J. \& Makeig, S. Information-based modeling of event-related brain dynamics. Prog. Brain Res. 159, 99-120 (2006).

82. Makeig, S., Debener, S., Onton, J. \& Delorme, A. Mining event-related brain dynamics. Trends Cogn. Sci. 8, 204-210 (2004).

83. Delorme, $A$, et al. EEGLAB, SIFT, NFT, BCILAB, and ERICA: new tools for advanced EEG processing. Comput. Intell. Neurosci. 2011, 130714 (2011).

84. Klimesch, W., Freunberger, R. \& Sauseng, P. Oscillatory mechanisms of process binding in memory. Neurosci. Biobehav. Rev. 34, 1002-1014 (2010).

85. Klimesch, W., Sauseng, P. \& Hanslmayr, S. EEG alpha oscillations: the inhibition-timing hypothesis. Brain Res. Rev. 53, 63-88 (2007).

86. Halgren, E., Boujon, C., Clarke, J., Wang, C. \& Chauvel, P. Rapid distributed fronto-parieto-occipital processing stages during working memory in humans. Cereb. Cortex 12, 710-728 (2002).

87. Capotosto, P., Babiloni, C., Romani, G. L. \& Corbetta, M. Frontoparietal cortex controls spatial attention through modulation of anticipatory alpha rhythms. J. Neurosci. 29, 5863-5872 (2009).

88. Siegel, M., Donner, T. H. \& Engel, A. K. Spectral fingerprints of large-scale neuronal interactions. Nat. Rev. Neurosci. 13, 121-134 (2012).

89. Sporns, O. Structure and function of complex brain networks. Dialogues Clin. Neurosci. 15, 247-262 (2013).

90. Hardmeier, M. et al. Reproducibility of functional connectivity and graph measures based on the phase lag index (PLI) and weighted phase lag index (wPLI) derived from high resolution EEG. PLOS ONE 9, e108648 (2014).

91. Boersma, M. et al. Disrupted functional brain networks in autistic toddlers. Brain Connect. 3, 41-49 (2013).

92. van den Heuvel, M. P., Mandl, R. C., Stam, C. J., Kahn, R. S. \& Hulshoff Pol, H. E. Aberrant frontal and temporal complex network structure in schizophrenia: a graph theoretical analysis. J. Neurosci. 30, 15915-15926 (2010).

93. Ahmadlou, M., Adeli, H. \& Adeli, A. Graph theoretical analysis of organization of functional brain networks in ADHD. Clin. EEG Neurosci. 43, 5-13 (2012).

94. Barrat, A., Barthelemy, M., Pastor-Satorras, R. \& Vespignani, A. The architecture of complex weighted networks. Proc. Natl Acad. Sci. USA 101, 3747-3752 (2004).

95. Sporns, O. Contributions and challenges for network models in cognitive neuroscience. Nat. Neurosci. 17, 652-660 (2014).

96. Fraga Gonzalez, G. et al. Graph analysis of EEG resting state functional networks in dyslexic readers. Clin. Neurophysiol. 127, 3165-3175 (2016).

97. Cohen, J. Statistical Power Analysis for the Behavioral Sciences 2nd edn (Lawrence Erlbaum Associates, Hillsdale, New Jersey, 1988).

98. Simes, R. J. An improved Bonferroni procedure for multiple tests of significance. Biometrika 73, 751-754 (1986).

99. Barttfeld, P. et al. A big-world network in ASD: dynamical connectivity analysis reflects a deficit in long-range connections and an excess of short-range connections. Neuropsychologia 49, 254-263 (2011).

100. Nicol, R. M. et al. Fast reconfiguration of high-frequency brain networks in response to surprising changes in auditory input. J. Neurophysiol. 107, 1421-1430 (2012).

101. Rommel, A. S. et al. Commonalities in EEG spectral power abnormalities between women with $\mathrm{ADHD}$ and women with bipolar disorder during rest and cognitive performance. Brain Topogr. 29, 856-866 (2016).

102. Skirrow, C. et al. Normalisation of frontal theta activity following methylphenidate treatment in adult attention-deficit/hyperactivity disorder. Eur. Neuropsychopharmacol. 25, 85-94 (2015).

103. Cheung, C. H. et al. Neurophysiological correlates of attentional fluctuation in attention-deficit/hyperactivity disorder. Brain Topogr. 30, 320-332 (2017).

104. Mattfeld, A. T. et al. Brain differences between persistent and remitted attention deficit hyperactivity disorder. Brain 137, 2423-2428 (2014).

105. Clarke, A. R. et al. Effects of methylphenidate on EEG coherence in attentiondeficit/hyperactivity disorder. Int. J. Psychophysiol. 58, 4-11 (2005). 